\title{
PEAU DE TILAPIA: UNE AVANCÉE TECHNOLOGIQUE DANS LE TRAITEMENT DES BRÛLURES?
}

\section{RÉVISER L'ARTICLE}

CABRAL, Anna Julie Medeiros ${ }^{1}$, LIMA, Camila Araújo Novais², CRUZ FILHO, Eduardo Franco Correia ${ }^{3}$, SOARES, Gabriel Lucena de Carvalho ${ }^{4}$, ESPÍNOLA, Paulo Francisco Lucena de Araújo ${ }^{5}$, ALVES, Fernanda Araújo6, CRUZ, Ana Suzy de Góis Melo ${ }^{7}$

CABRAL, Anna Julie Medeiros. Et al. Peau de tilapia: une avancée technologique dans le traitement des brûlures?. Revista Científica Multidisciplinar Núcleo do Conhecimento. Année. 06, Ed. 12, vol. 05, p. 50 à 64. Décembre 2021. ISSN : 24480959, Lien d'accès: https://www.nucleodoconhecimento.com.br/sante/peau-detilapia, DOI: 10.32749/nucleodoconhecimento.com.br/sante/peau-de-tilapia

\section{RÉSUMÉ}

Introduction: La brûlure est l'une des plus grandes agressions que le corps puisse subir. L'approche varie en fonction du degré de brûlure, depuis l'utilisation de chlorhexidine, de sulfadiazine d'argent, de débridement de tissu nécrotique, de pansements biosynthétiques et de peaux artificielles. Cependant, ces deux derniers ont des coûts élevés, de sorte que plusieurs études ont émergé dans le but de rechercher des options plus viables, telles que l'utilisation de la peau de tilapia du Nil dans les brûlures, en raison de ses propriétés curatives. Cela dit, le présent article a

\footnotetext{
${ }^{1}$ Étudiant du cours de médecine du Centre universitaire de João Pessoa - UNIPÊ, João Pessoa - PB. ORCID: 0000-0002-5212-5521.

${ }^{2}$ Étudiant du cours de médecine du Centre universitaire de João Pessoa - UNIPÊ, João Pessoa - PB. ORCID: 0000-0003-4609-4260.

${ }^{3}$ Étudiant du cours de médecine du Centre universitaire de João Pessoa - UNIPÊ, João Pessoa - PB. ORCID: 0000-0001-6684-2161.

${ }^{4}$ Étudiant du cours de médecine du Centre universitaire de João Pessoa - UNIPÊ, João Pessoa - PB. ORCID: 0000-0002-0618-4037.

${ }^{5}$ Étudiant du cours de médecine du Centre universitaire de João Pessoa - UNIPÊ, João Pessoa - PB. ORCID: 0000-0001-7964-2118.

${ }^{6}$ Conseiller d'orientation. ORCID: 0000-0001-5235-5020.

${ }^{7}$ Conseiller. ORCID: 0000-0002-0668-0540.
}

RC: 104336

Disponible en: https://www.nucleodoconhecimento.com.br/sante/peau-de-tilapia 
pour guide une question: cette nouvelle méthode est-elle, en fait, une avancée technologique aussi importante pour le traitement des patients brûlés qu'elle semble l'être? Objectif: Analyser l'utilisation de la peau du tilapia du Nil chez les patients souffrant de brûlures, ainsi que comparer avec d'autres techniques préétablies. Méthodes: II s'agit d'une revue bibliographique intégrative avec une approche qualitative. Les données ont été recueillies par le biais de bases de données PubMed et de la bibliothèque virtuelle de santé (BVS), de 2015 à 2020. Résultats: Les articles indiquent un bon pronostic à l'utilisation de la peau de tilapia du Nil par rapport aux autres options en vigueur pour le traitement des brûlures, avec un avantage significatif dans la réduction du nombre de pansements nécessaires, pour une meilleure adhérence à la plaie. De plus, il présente des caractéristiques microscopiques similaires à celles de la peau humaine, telles qu'une résistance élevée à la traction et une extension de la rupture, réduisant le temps de réépithélialisation et l'intensité de la douleur, ainsi que les coûts de traitement. Conclusion: Compte tenu des résultats de la littérature rapportés dans la présente revue, il est conclu que les études avec la peau de tilapia du Nil s'avèrent être une modalité révolutionnaire avec des avantages dans le traitement des patients présentant des lésions cutanées superficielles et profondes. Par conséquent, les chercheurs ont conclu, en répondant à la question guide, que la nouvelle méthode est, oui, une avancée importante dans le domaine du traitement des brûlures, car son employabilité est confirmée, en plus de démontrer un avantage sur certaines des principales alternatives préexistantes.

Mots-clés: Tilapia, Brûlures, Pansements biologiques.

\section{INTRODUCTION}

La vitesse et le mode de vie imposés par la modernité font augmenter l'incidence du nombre de brûlures aujourd'hui. Bien au-delà des blessures physiques, les brûlures sont également responsables de problèmes économiques associés à des dommages irréparables pour les patients et leurs familles (HU et al., 2017). La brûlure est l'une des plus grandes agressions que l'organisme humain peut subir, et on estime qu'au 
Brésil, il y a environ 1 million d'accidents de brûlure chaque année, selon Leontsinis et al. (2018), s'il s'agit d'un simple coup de chaleur, de brûlures au premier degré, jusqu'à la destruction totale de la peau (épiderme et derme) et des tissus adjacents, comme dans les brûlures au troisième degré (ALVES et al., 2015).

Lorsque nous considérons le traitement des brûlures au Brésil, nous sommes confrontés à deux réalités: celle du réseau public et privé. Dans les centres de traitement du réseau public, la conduite suivie en ce qui concerne les pansements dans les lésions du deuxième degré est le bain quotidien avec $2 \%$ de chlorhexidine et le pansement est fait avec la antimicrobien topique argent sulfadiazine à $1 \%$ jusqu'à réparation complète de la lésion; tandis que dans les brûlures au troisième degré, le tissu nécrotique est débridé en plusieurs étapes, puis le pansement avec de la sulfadiazine d'argent à $1 \%$ est effectué jusqu'à ce que le lit de la plaie soit préparé pour la greffe. D'autre part, dans le réseau privé, en tenant compte des différentes conditions financières de chaque patient, des pansements biosynthétiques et des peaux artificielles, des options importées et coûteuses peuvent être utilisées (ALVES et al., 2015).

Cependant, en analysant la réalité vécue en Europe et aux États-Unis, le traitement implique souvent l'utilisation de peaux homologues (utilisant des banques de peau), d'hétérologues (d'origine animale), de pansements biosynthétiques et de dermes artificiels (ALVES et al., 2015). Au Brésil, il y a encore quelques confrontations pour parvenir à la même praticité que ces autres pays. Culturellement, il y a encore une grande résistance au don de peau, ce qui finit par limiter la disponibilité du tissu pour répondre à la grande demande. En outre, en prenant comme référence le ministère de la Santé, afin que le Brésil puisse répondre au besoin de peau homologue pour une utilisation dans les brûlures, il serait nécessaire d'avoir 13 banques de peau réparties sur tout le territoire national, mais il n'y en a que cinq (São Paulo, Curitiba, Rio de Janeiro, Porto Alegre et Recife - qui est handicapé). En plus de ces limitations, le Brésil n'a jamais eu de peau d'animal enregistrée auprès de l'Agence nationale de surveillance de la santé (ANVISA) et mise à disposition par le Système 
unifié de santé (SUS), qui finit également par restreindre l'utilisation de la peau hétérologuechez les patients brûlés (LEONTSINIS et al., 2018).

Ainsi, plusieurs études ont émergé dans le but de rechercher des pansements à utiliser chez les patients brûlés. Ces pansements visent à réduire les effets de la contamination sur les lésions, favorisent le processus de guérison et offrent de meilleurs résultats esthétiques. Ayant, idéalement, une bonne flexibilité et adhérence au lit, une résistance aux étirements, une manipulation facile, une capacité à supprimer la douleur, un faible coût et facile à obtenir, ainsi que prévient les pertes hydroélectrolytiques, la contamination bactérienne, favorise l'épithélialisation des brûlures et fournit la formation de tissu de granulation adéquat. L'une des options est la recherche de substituts cutanés temporaires et de pansements de matériaux synthétiques ou biosynthétiques, car ils réduisent la fréquence des changements de pansement, cependant, les coûts élevés et son inefficacité pour les brûlures profondes ont stimulé la recherche de matériaux biologiques comme alternatives viables (COSTA et al., 2019; MARCELO et BRANDT, 2019).

Dans ce contexte, en 2011, le chirurgien plasticien marcelo Borges, après avoir vu un article dans le Jornal do Commercio de Pernambuco parlant de l'utilisation de la peau de l'espèce Tilapia du Nil (Oreochromis niloticus) dans l'artisanat, l'un des poissons les plus cultivés au Brésil, a d'abord eu l'idée d'utiliser cette peau dans le traitement des brûlures. Mais ce n'est qu'en 2014 qu'il a partagé l'idée avec le chirurgien plasticien du Ceará Edmar Maciel, puis a commencé des études sur I'utilisation de la peau du Tilapia du Nil dans les brûlures. Le chercheur Odorico Moraes, président-directeur du Centre de recherche et de développement de médicaments (NPDM), à l'Université fédérale du Ceará (UFC), et le chirurgien plasticien de Goiás Nelson Piccolo (LIMA JUNIOR et al.), ont également été intégrés pour constituer la coordination des travaux. 2017).

Comme l'a raconté Lima Junior (2017), la peau du tilapia du Nil montre un épiderme recouvert d'un épithélium stratifié, suivi de vastes couches de collagène. Grâce à sa caractéristique de guidage et de définition de la plupart des tissus, il est configuré comme un excellent composant des biomatériaux, en plus de permettre la 
biodégradabilité et la biocompatibilité, ce qui favorise son application (LIMA JUNIOR, 2017; LIMA JUNIOR et al., 2019b, 2020b).

Cela dit, la bonne question est : " Cette nouvelle méthode est-elle, en fait, une avancée technologique aussi importante pour le traitement des patients brûlés qu'elle semble l'être ? " L'objectif est donc, à travers cette étude, d'analyser si la peau du tilapia du Nil est une alternative viable à utiliser chez les patients souffrant de brûlures, ainsi que de comparer avec d'autres techniques déjà utilisées, en vérifiant les avantages de l'adoption de cette nouvelle méthode.

Plus précisément, cette étude vise à démontrer la bonne adhérence des pansements cutanés au tilapia dans le traitement des brûlures, en corrélant les propriétés physico-chimiques du tilapia avec les bénéfices du pansement et en exposant la non-infériorité des pansements cutanés au tilapia par rapport aux autres solutions sur le marché. En plus d'inciter à poursuivre les recherches scientifiques pour obtenir des résultats plus solides sur le sujet.

\section{MÉTHODOLOGIE}

II s'agit d'une revue de littérature intégrative, avec une approche qualitative, dans un but descriptif d'études nationales et internationales. En outre, il a un caractère appliqué, avec l'utilisation d'une procédure bibliographique pour l'analyse des données respectives collectées liées à l'utilisation de la peau de tilapia chez les patients brûlés.

La présente étude a été réalisée par la recherche d'articles grâce à l'accès aux bases de données pubmed et à la Bibliothèque virtuelle de santé (BVS). Dans un premier temps, les descripteurs pertinents pour le thème choisi ont été recueillis, à savoir le vocabulaire des descripteurs en sciences de la santé (DeCS). Les termes choisis étaient «Tilapia ", " brûlures » et "Pansements biologiques » dans les langues: portugais, anglais et espagnol. Ces descripteurs ont été associés aux opérateurs booléens «OR » et “ $A N D$ » en élaborant la formule de recherche suivante: « (Tilápia OR Tilapia OR Tilapia) AND (Queimaduras OR Burns OR 
Quemaduras) AND (Curativos Biológicos OR Biological Dressings OR Apósitos Biológicos) ».

Ainsi, en appliquant la logique de la formule susmentionnée, un total de 31 articles ont été trouvés dans les bases de données sélectionnées, 14 articles publiés et 17 disponibles dans la BVS. Les critères d'inclusion étaient les suivants : articles disponibles dans leur intégralité, qui décrivaient le thème lié à la revue intégrative et articles publiés et indexés dans les bases de données publiées au cours des années 2015, 2016, 2017, 2018, 2019, 2020 et 2021, qui présentaient la langue en anglais, portugais et espagnol. De plus, les articles qui ont été dupliqués, qui n'avaient pas un contenu complet et qui ne répondaient pas aux objectifs de recherche ont été exclus de la revue intégrative.

La revue bibliographique intégrative n'est pas pratique, par conséquent, elle ne nécessite pas de soumission au Comité d'éthique de la recherche (CEP). Dans cette perspective, un tableau a été élaboré montrant les principales informations des bibliographies utilisées, présentant les données se référant au titre, aux auteurs, à l'année de publication, au pays et aux principaux résultats de chacune.

\section{RÉSULTATS}

Tableau 1 : Utilisation de la peau de tilapia dans les brûlures : fondement théorique et implications pratiques.

\begin{tabular}{|l|l|l|l|}
\hline & Titre & $\begin{array}{l}\text { Auteurs, } \\
\text { année de } \\
\text { publication } \\
\text { et pays }\end{array}$ & \\
\hline ARTICLE & $\begin{array}{l}\text { Uso da pele de tilápia } \\
\text { (Oreochromis niloticus), } \\
\text { como curativo biológico } \\
\text { oclusivo, no tratamento de } \\
\text { queimaduras }\end{array}$ & et al., 2017, de tilapia a une adhérence \\
\hline 1 & Brasil & $\begin{array}{l}\text { considérable aux plaies } \\
\text { brûlées chez les rats d'essai, } \\
\text { avec des résultats positifs }\end{array}$ \\
\hline
\end{tabular}




\begin{tabular}{|c|c|c|c|}
\hline & & & $\begin{array}{l}\text { pendant la cicatrisation, sans } \\
\text { mutations significatives dans } \\
\text { les domaines biochimique et } \\
\text { hématologique de la fonction } \\
\text { hépatique et rénale, } \\
\text { constituant une possibilité de } \\
\text { pansement biologique. }\end{array}$ \\
\hline $\begin{array}{l}\text { ARTICLE } \\
2\end{array}$ & $\begin{array}{l}\text { Xenoenxerto (pele da } \\
\text { Tilápia-do-Nilo) e hidrofibra } \\
\text { com prata no tratamento } \\
\text { das queimaduras de II } \\
\text { grau em adultos }\end{array}$ & $\begin{array}{lr}\text { De } & \text { Miranda } \\
\text { e } & \text { Brandt, } \\
\text { 2019, } & \text { Brasil }\end{array}$ & $\begin{array}{l}\text { La recherche a montré que } \\
\text { le tilapia du Nil est efficace } \\
\text { dans la fonction de } \\
\text { pansement biologique } \\
\text { occlusif dans le traitement } \\
\text { des brûlures au deuxième } \\
\text { degré chez l'adulte, avec des } \\
\text { moyens similaires en ce qui } \\
\text { concerne le temps de } \\
\text { traitement, la référence de la } \\
\text { douleur et le besoin de } \\
\text { remplacement, en ce qui } \\
\text { concerne la prise en charge } \\
\text { des patients atteints } \\
\text { d'Aquacel } A G \circledast \text {. }\end{array}$ \\
\hline $\begin{array}{l}\text { ARTICLE } \\
3\end{array}$ & $\begin{array}{l}\text { Avaliação microscópica, } \\
\text { estudo histoquímico e } \\
\text { análise de propriedades } \\
\text { tensiométricas da pele de } \\
\text { tilápia do Nilo }\end{array}$ & $\begin{array}{l}\text { Alves et al., } \\
\text { 2015, Brasil }\end{array}$ & $\begin{array}{l}\text { La peau de tilapia présente } \\
\text { des faisceaux de fibres de } \\
\text { collagène denses, } \\
\text { principalement de type 1, } \\
\text { présentant des } \\
\text { caractéristiques } \\
\text { microscopiques similaires à } \\
\text { celles de la peau humaine, } \\
\text { ainsi qu'une résistance }\end{array}$ \\
\hline
\end{tabular}




\begin{tabular}{|c|c|c|c|}
\hline & & & $\begin{array}{l}\text { élevée à la traction et une } \\
\text { extension à la rupture, ce qui } \\
\text { la rend prometteuse en tant } \\
\text { que biomatériau en } \\
\text { médecine régénérative. }\end{array}$ \\
\hline $\begin{array}{l}\text { ARTICLE } \\
4\end{array}$ & $\begin{array}{l}\text { Innovative Burn Treatment } \\
\text { Using Tilapia Skin as a } \\
\text { Xenograft: A Phase II } \\
\text { Randomized Controlled } \\
\text { trial }\end{array}$ & $\begin{array}{l}\text { Lima Júnior } \\
\text { et al., 2020, } \\
\text { Brasil }\end{array}$ & $\begin{array}{l}\text { L'essai clinique randomisé a } \\
\text { utilisé un échantillon de } 62 \\
\text { participants et a démontré } \\
\text { qu'il y avait une réduction de: } \\
\text { le temps de } \\
\text { réépithélialisation, l'intensité } \\
\text { de la douleur, la quantité } \\
\text { d'esthétique / analgésiques } \\
\text { et la nécessité de changer } \\
\text { de pansements par rapport } \\
\text { au groupe témoin, par } \\
\text { conséquent, la peau de } \\
\text { tilapia présentait des } \\
\text { avantages. }\end{array}$ \\
\hline $\begin{array}{l}\text { ARTICLE } \\
5\end{array}$ & $\begin{array}{l}\text { Tratamento de } \\
\text { queimaduras de segundo } \\
\text { grau profundo em } \\
\text { abdômen, coxas e } \\
\text { genitália: uso da pele de } \\
\text { tilápia como um } \\
\text { xenoenxerto }\end{array}$ & $\begin{array}{l}\text { Lima Júnior } \\
\text { et al., 2020, } \\
\text { Brasil }\end{array}$ & $\begin{array}{l}\text { Le rapport de cas a contribué } \\
\text { à réduire les limitations liées } \\
\text { à l'accès à des zones } \\
\text { anatomiques plus } \\
\text { inaccessibles à utiliser, } \\
\text { présentant de bons résultats } \\
\text { dans l'application dans les } \\
\text { organes génitaux et la région } \\
\text { inguinale. C'est une option } \\
\text { de haute disponibilité et } \\
\text { d'application simple. }\end{array}$ \\
\hline
\end{tabular}

ARTICLE Marine Collagen Peptides Zhang Hu et Le test de grattage in vitro et 


\begin{tabular}{|c|c|c|}
\hline $\begin{array}{l}\text { from the Skin of Nile } \\
\text { Tilapia (Oreochromis } \\
\text { niloticus): Characterization } \\
\text { and Wound Healing } \\
\text { Evaluation }\end{array}$ & $\begin{array}{l}\text { al., 2017, } \\
\text { China }\end{array}$ & $\begin{array}{l}\text { in vivo a révélé de } \\
\text { conséquences significative } \\
\text { dans la fermeture } \\
\text { grattage avec la peau d } \\
\text { tilapia, avec une évolution d } \\
\text { processus de guérison pa } \\
\text { échaudure d'épaisse } \\
\text { partielle profonde chez le } \\
\text { lapins testés, se présental } \\
\text { comme un traitemer } \\
\text { prometteur. }\end{array}$ \\
\hline
\end{tabular}

ARTICLE A

Comparison

Randomized

Lyophilized Study

of

Lima Júnior,

Cet

Cette étude pilote a Skin and SilverImpregnated Sodium Carboxymethylcellulose for the Treatment of Superficial PartialThickness Burns

\section{ARTICLE} 8

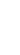 d'infériorité à la peau de tilapia lyophilisée en tant qu'option thérapeutique dans les brûlures, par rapport au pansement à la carboxyméthylcellulose sordide imprégné d'argent, avec efficacité et innocuité, permettant le développement d'essais cliniques randomisés plus complexes à l'avenir. démontré quill ny a pas

\begin{tabular}{|lr|l|l|} 
Elaboração de & um & Leontsinis et & L'article a révélé l'importance \\
protocolo & para & al., 2018, & d'élaborer et de mettre en \\
implementação & e & Brasil & $\begin{array}{l}\text { œuvre des protocoles pour la } \\
\text { première banque de peau }\end{array}$ \\
funcionamento do primeiro & & $\begin{array}{l}\text { d'animaux aquatiques au } \\
\text { banco de pele animal do }\end{array}$ & \\
Brasil: $\quad$ Relato de & & monde, renforçant ainsi la
\end{tabular}


experiência

ARTICLE Use of Tilapia Skin as a

9 Xenograft for Pediatric 2019, Burn Treatment: A Case Estados Report Unidos nécessité d'établir la

normalisation du système.
Costa et al., La xénogreffe de peau de tilapia dans le rapport de cas d'une victime de brûlures de 3 ans a montré des résultats positifs avec un impact social et financier significatif sur le système de santé.

ARTICLE Innovative treatment using Lima Júnior 10 tilapia skin as a xenograft for partial thickness burns Inglaterra after a gunpowder explosion

Le rapport de cas dépeint un patient de 23 ans présentant des brûlures superficielles et profondes sur le membre supérieur droit et gauche, respectivement. Avec l'utilisation de la peau de tilapia, la réépithélialisation a été effectuée en peu de temps, sans effets secondaires et nécessitant un changement de pansement.

ARTICLE Pediatric Burn Treatment Lima Júnior, L'utilisation de la peau de 11 Using Tilapia Skin as a 2020, Xenograft for Superficial Inglaterra Partial-Thickness Wounds: A Pilot Study tilapia a été utilisée comme ressource supplémentaire avec la sulfadiazine d'argent. L'étude a révélé qu'en plus d'apporter des avantages aux patients, elle a réduit les coûts de traitement, ainsi que pour les professionnels 
ARTICLE Comprehensive

12 de la santé, comme la réduction de la charge de travail.

$\mathrm{Ge}$ et al., Le pansement en hydrogel 2020, China de collagène a montré la promotion de la formation de couches épidermiques et la maturation des appendices cutanés, démontrant qu'il s'agissait d'un excellent nouveau pansement efficace dans la gestion des brûlures profondes.

ARTICLE Tecnologias inovadoras: Lima Júnior, La peau de tilapia dans les 13 uso da pele da tilápia do 2017, Brasil brûlures est un brevet Nilo no tratamento de brésilien, et l'objectif de la queimaduras e feridas recherche est l'enregistrement de la peau auprès de l'Agence nationale de surveillance de la santé (ANVISA) et mis à disposition par le système de santé unifié (SUS).

ARTICLE Chitosan hydrogel in Ouyang et Les peptides marins extraits 14 combination with marine al. 2018, du tilapia ont montré une peptides from tilapia for China composition semblable à burns healing celle du collagène, en plus d'une activité antibactérienne significative. II présente une efficacité de guérison élevée et des compétences de 


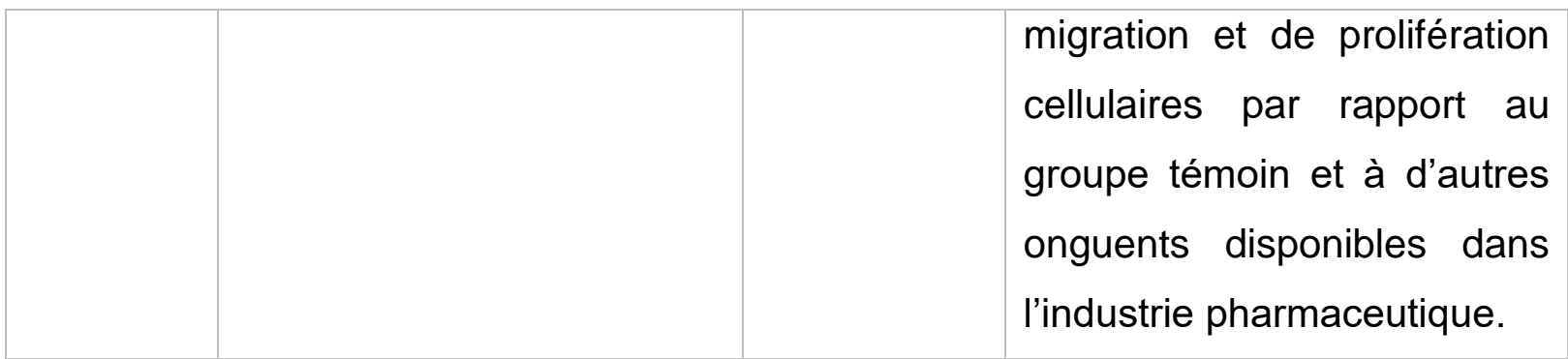

Source: Développé par l'auteur (2021)

La revue comprenait 14 articles, parmi lesquels, deux (14,3\%) ont rapporté les résultats à l'aide de tests sur des animaux, 14,3\% sur une analyse comparative entre les traitements traditionnels et l'utilisation de la peau de tilapia, et $21,4 \%$ sur les mécanismes de composition et les propriétés par rapport à la peau humaine. L'utilisation d'essais randomisés représentait 7,1\% des articles, $28,6 \%$ présentaient les résultats dans des rapports de cas et $14,3 \%$ expliquaient le développement de la technique jusqu'à son importance dans les banques de peau de nos jours.

L'étude menée au Centre de traitement des brûlures de l'Institut Dr José Frota, hôpital public de Fortaleza, Ceará, Brésil, a sélectionné 62 patients hospitalisés entre octobre 2016 et septembre 2017, qui ont été victimes de brûlures. Les variantes évaluées étaient: le nombre de fois qu'il y avait un échange du pansement occlusif et des jours pour la cicatrisation des plaies, l'utilisation d'analgésiques ou d'anesthésiques, la sensibilité douloureuse, l'amélioration de la brûlure dans le retrait du pansement. L'étude a analysé les données et divisé les participants, de: sexe, âge, agents responsables de l'accident, surface corporelle et segments brûlés, et pour cela, divisé en trois classifications, A - patients ambulatoires avec des brûlures superficielles partielles inférieures à 10\%, B - patients hospitalisés avec les mêmes 10 à 20\%, C - patients hospitalisés avec des brûlures profondes d'épaisseur partielle de 5 à $15 \%$, et chacune de ces classifications a été divisée au hasard en deux groupes de traitement : 48,4\% $(n=30)$ Groupe test utilisant la peau de tilapia comme xénogreffe et $51,6 \%(n=32)$ Groupe témoin : conventionnel avec de la crème de sulfadiazine. Le principal mécanisme de brûlure du groupe test (classifications $\mathrm{A}, \mathrm{B}$ et $\mathrm{C}$ utilisant la peau de tilapia comme traitement) était par 
liquides chauds $56,25 \%(n=18)$. L'intensité de la douleur était plus faible dans le groupe test que dans le groupe témoin. Le nombre de pansements requis pendant le traitement, dans le groupe d'essai, il était plus faible, présentant $2,08 \pm 0,28(A), 2,33$ $\pm 0,71(B), 6,10 \pm 2,02(C)$, tandis que dans le groupe témoin 5,80 $\pm 0,42(A), 11,00 \pm$ 0,47 (B), 20,20 $\pm 1,69$ (C) en tenant compte de la valeur $p$ de 0,0001. En ce qui concerne le nombre de jours pour une réépithélialisation complète, le groupe test a présenté les meilleurs résultats, avec : 9,77 $\pm 0,83(A), 10,56 \pm 1,13(B), 18,10 \pm 0,99$ (C), tandis que le groupe témoin : 11,20 \pm 0,63 (A), 11,70 $\pm 0,67$ (B), 21,30 $\pm 1,42 @$, considérant environ $\mathrm{P}<0.0147$ (LIMA JUNIOR et al., 2020a)

Une autre étude menée au Centre de traitement des brûlures de Fortaleza, au Ceará, au Brésil, a sélectionné 30 enfants, âgés de 2 à 12 ans, hospitalisés entre mai 2017 et mars 2018. Les variables utilisées étaient : le nombre total de jours pour la guérison complète de la brûlure et le nombre de pansements utilisés. Les patients ont été divisés en deux groupes, dans lesquels $50 \%$ ont été ascensionnés vers le groupe de test cutané tilapia et le reste vers le groupe de sulfadiazine d'argent. Le nombre moyen de jours pour terminer la réépithélialisation était de 10,47 $\pm 0,74$ dans le groupe de la sulfadiazine d'argent et de 10,07 $\pm 0,46$ dans le groupe de la peau du tilapia. La différence entre les deux groupes n'était pas statistiquement significative $(P=0,0868)$. Le nombre de pansements sous anesthésie effectués chez les patients traités par la peau de tilapia était inférieur à celui effectué chez les volontaires traités par la sulfadiazine d'argent. Une différence statistiquement significative a également été trouvée pour le nombre de pansements sans anesthésiques. Enfin, le nombre total de pansements a été significativement réduit dans le groupe cutané du tilapia $(3,00 \pm 0,76)$ par rapport au groupe de la sulfadiazine d'argent $(9,27 \pm 1,39)$ (LIMA JUNIOR et al., 2019a).

\section{DISCUSSION}

L'étude des propriétés cutanées du tilapia est un bon point de départ pour comprendre son potentiel d'utilisation dans les brûlures. Pour cela, il convient d'analyser d'abord les résultats de $\mathrm{Hu}$ et al. (2017) qui a étudié l'utilisation d'un 
concentré de polypeptide de collagène extrait de la peau du tilapia, en se concentrant sur son potentiel curatif. Des essais in vitro utilisant des cellules humaines préparées ont montré que l'ajout de $50,0 \mu \mathrm{g} / \mathrm{mL}$ de concentré au tissu lésé apportait des résultats statistiquement significatifs en ce qui concerne la vitesse de régénération et de réépithélialisation du tissu. D'autre part, les études in vivo du même auteur ont montré des résultats prometteurs concernant la guérison par échaudage partiel profond chez les lapins testés. Les résultats étaient similaires à ceux obtenus avec le pansement à base d'hydrogel de collagène, étudié par Ge et al. (2020), pour les brûlures profondes.

En outre, d'autres études ont été réalisées pour évaluer les propriétés de la peau de l'animal. Selon Alves et al. (2015), la peau de tilapia présente des faisceaux de fibres de collagène denses, principalement de type 1, avec des caractéristiques microscopiques similaires à celles de la peau humaine, ainsi qu'une résistance à la traction et une extension élevées, ce qui la rend prometteuse en tant que biomatériau en médecine régénérative. L'étude d'Ouyang et al. (2018) corrobore ces résultats, ainsi que ceux de $\mathrm{Hu}$ et al. (2017), qui aborde également l'activité antibactérienne du matériau et compromet son efficacité de durcissement avec d'autres solutions disponibles sur le marché, obtenant une efficacité élevée, en plus de fournir la migration et la prolifération cellulaire.

Validé ce potentiel curatif de la peau de tilapia, il est nécessaire de comprendre la facilité d'utilisation de ce matériau sous forme de pansements biologiques. Selon l'étude de Lima Junior (2017), la peau de tilapia a une adhérence considérable aux brûlures chez les rats d'essai, avec des résultats positifs pendant la guérison, sans mutations significatives dans les domaines biochimique et hématologique de la fonction hépatique et rénale, constituant une possibilité positive en tant que pansement biologique. Corroborant ces résultats, l'essai clinique randomisé de Lima Junior et al. (2020c) ont montré une bonne adhérence chez les patients, ainsi que d'autres avantages par rapport à d'autres pansements, tels que Aquacel $\mathrm{Ag} \circledast$, qui seront discutés plus tard. 
Cela dit, il vaut la peine de comparer la peau du tilapia avec d'autres solutions existantes en matière de brûlures. Lima Junior et al. (2020c) et Marcelo et Brandt (2019) ont promu des études qui ne montrent aucune infériorité dans la peau de tilapia lyophilisé, par rapport au pansement sordide à la carboxyméthylcellulose imprégné d'argent, Aquacel Ag ${ }^{\circledR}$. L'étude de Lima Junior et al. (2020c) souligne que l'utilisation de la peau de tilapia offre une amélioration subjective de la quantité de douleur signalée et du nombre de pansements nécessaires, réduisant ainsi le coût du traitement et la charge de travail de l'équipe. Par conséquent, la peau de tilapia est une bonne option pour le traitement des brûlures.

\section{CONCLUSION}

Compte tenu des résultats de la littérature rapportés dans la présente revue, il est conclu que des études avec la peau du tilapia du Nil, des poissons d'eau douce, menées par des chercheurs de l'Université fédérale du Ceará, qui ont été, depuis 2016, les pionniers à breveter la méthode. De plus, il s'agit d'une modalité révolutionnaire avec de nombreux avantages dans le traitement des patients présentant des lésions cutanées superficielles et profondes s'étendant à plusieurs domaines de la médecine, testés en médecine vétérinaire, à l'intérieur et à l'extérieur du pays (LIMA JUNIOR, 2017).

Sur la base des dossiers de recherche, les avantages de l'utilisation de Peau de tilapia dans le traitement des brûlures sont renforcés, étant donné qu'il offre une réduction de la douleur du patient en raison de la plus longue période d'adhésion à la peau du patient. En plus du pouvoir réduit d'infection et de contamination, une résistance élevée en raison de la grande quantité de collagène présente dans la peau de poisson, ce qui entraîne une bonne esthétique et un faible coût, par rapport à d'autres formes de traitement.

Par conséquent, les chercheurs sont arrivés à la conclusion, en répondant à la question guide, que la nouvelle méthode est, oui, une avancée importante dans le traitement des brûlures, car son employabilité est confirmée, en plus de démontrer un avantage sur certaines des principales alternatives préexistantes. 


\section{RÉFÉRENCES}

ALVES, Ana; VERDE, Maria; FERREIRA FILHO, Antônio; SILVA, Paulo; FEITOSA, Victor; LIMA JUNIOR, Edmar; MIRANDA, Marcelo; MORAES FILHO, Manoel. Avaliação microscópica, estudo histoquímico e análise de propriedades tensiométricas da pele de tilápia do Nilo. Revista Brasileira de Queimaduras, v. 14, n. 3, p. 203-210, 2015.

COSTA, Bruno; LIMA JÚNIOR, Edmar; MORAES FILHO, Manoel; FECHINE, Francisco; MORAES, Maria; SILVA JUNIOR, Francisco; SOARES, Maria; ROCHA, Marina. Use of Tilapia Skin as a Xenograft for Pediatric Burn Treatment: A Case Report. Journal of Burn Care \& Research, v. 40, n. 5, p. 714-717, 2019.

GE, Baosheng; WANG, Haonan; LI, Jie; LIU, Hengheng; YIN, Yonghao; ZHANG, Naili; QIN, Song. Comprehensive Assessment of Nile Tilapia Skin (Oreochromis niloticus) Collagen Hydrogels for Wound Dressings. Marine Drugs, v. 18, n. 4, p. 178, 2020.

HU, Zhang; YANG, Ping; ZHOU, Chunxia; LI, Sidong; HONG, Pengzhi. Marine Collagen Peptides from the Skin of Nile Tilapia (Oreochromis niloticus): Characterization and Wound Healing Evaluation. Marine Drugs, v. 15, n. 4, p. 102, 2017.

LEONTSINIS, Cybele; LIMA JUNIOR, Edmar; MORAIS FILHO, Manoel; BRITO, Maria; ROCHA, Marina; NASCIMENTO, Maria; SILVA JUNIOR, Francisco; MIRANDA, Marcelo. Elaboração de um protocolo para implementação e funcionamento do primeiro banco de pele animal do Brasil: Relato de experiência. Revista Brasileira de Queimaduras, v. 17, n. 1, p. 66-71, 2018.

LIMA JUNIOR, Edmar; PICOLLO, Nelson; MIRANDA, Marcelo; RIBEIRO, Wesley; ALVES, Ana; FERREIRA, Guilherme; PARENTE, Ezequiel, MORAES FILHO, Manoel. Uso da pele de tilápia (Oreochromis niloticus), como curativo biológico 
oclusivo, no tratamento de queimaduras. Revista Brasileira de Queimaduras, v. 16, n. 1, p. 10-17, 2017.

LIMA JÚNIOR, Edmar. Tecnologias inovadoras: uso da pele da tilápia do Nilo no tratamento de queimaduras e feridas. Revista Brasileira de Queimaduras, v. 16, n. 1, p. 1-2, 2017.

LIMA JÚNIOR, Edmar; MORAES FILHO, Manoel; FORTE, Antônio; COSTA, Bruno; ; FECHINE, Francisco; ALVES, Ana; MORAES, Maria; ROCHA, Marina; SILVA JUNIOR, Francisco; SOARES, Maria; BEZERRA, Alane; MARTINS, Camila; MATHOR, Mônica. Pediatric Burn Treatment Using Tilapia Skin as a Xenograft for Superficial Partial-Thickness Wounds: A Pilot Study. Journal of Burn Care \& Research, 2019a.

LIMA JUNIOR, Edmar; MORAES FILHO, Manoel; COSTA, Bruno Almeida; FECHINE, Francisco; MORAES, Maria; SILVA JUNIOR, Francisco; SOARES, MARIA; ROCHA, MARINA, LEONTSINIS, Cybele. Innovative treatment using tilapia skin as a xenograft for partial thickness burns after a gunpowder explosion. Journal of Surgical Case Reports, v. 2019, n. 6, $2019 \mathrm{~b}$.

LIMA JÚNIOR, Edmar; MORAES FILHO, Manoel; COSTA, Bruno; ROHLEDER, Andréa; ROCHA, Marina; FECHINE, Francisco; FORTE, Antonio. ALVES, Ana, SILVA JUNIOR, Francisco; MARINS, Camila; MATHOR, Mônica; MORAES, Maria. Innovative Burn Treatment Using Tilapia Skin as a Xenograft: A Phase II Randomized Controlled Trial. Journal of Burn Care \& Research, v. 41, n. 3, p. 585592, 2020a.

LIMA JUNIOR, Edmar; MORAES, Manoel Odorico; COSTA, Bruno Almeida; UCHÔA, Alex; MARTINS, Camila; MORAES, Maria; ROCHA, Marina; FECHINE, Rodrigo. Treatment of deep second-degree burns on the abdomen, thighs, and genitalia: use of tilapia skin as a xenograft. Revista Brasileira de Cirurgia Plástica (RBCP) - Brazilian Journal of Plastic Sugery, v. 35, n. 2, p. 243-248, 2020b. 
LIMA JÚNIOR, Edmar; MORAES FILHO, Manoel ; COSTA, Bruno Almeida; FECHINE, Francisco; ROCHA, Marina; VALE, Mariana; DIÓGENES, Ana; UCHÔA, Alex; SILVA JUNIOR, Francisco; MARTINS, Camila; BANDEIRA, Tereza; RODRIGUES, Felipe; PAIER, Carlos; MORAES, Maria. A Randomized Comparison Study of Lyophilized Nile Tilapia Skin and Silver-Impregnated Sodium Carboxymethylcellulose for the Treatment of Superficial Partial-Thickness Burns. Journal of Burn Care \& Research, v. 42, n. 1, p. 41-48, 2020 c.

DE MIRANDA, Marcelo José Borges; BRANDT, Carlos Teixeira. Xenoenxerto (pele da Tilápia-do-Nilo) e hidrofibra com prata no tratamento das queimaduras de II grau em adultos. Rev. bras. cir. plást, p. 79-85, 2019.

OUYANG, Qian-Qian; HU, Zhang; LIN, Zhen-Peng; QUAN, Wei-Yan; DENG, YiFeng; LI, Si-Dong; LI, Pu-Wang, CHEN, Yu. Chitosan hydrogel in combination with marine peptides from tilapia for burns healing. International Journal of Biological Macromolecules, v. 112, p. 1191-1198, 2018.

Déposée: Juillet 2021.

Approbation : Décembre 2021. 